\title{
Treatment of Posttraumatic Stress Disorder by Trained Lay Counselors in an African Refugee Settlement: A Randomized Controlled Trial
}

\author{
Frank Neuner \\ University of Konstanz and vivo Germany
}

\author{
Patience Lamaro Onyut \\ Vivo Uganda and Mbarara University of Science and \\ Technology
}

\author{
Verena Ertl, Michael Odenwald, Elisabeth Schauer, and Thomas Elbert \\ University of Konstanz and vivo Germany
}

\begin{abstract}
Traumatic stress due to conflict and war causes major mental health problems in many resource-poor countries. The objective of this study was to examine whether trained lay counselors can carry out effective treatment of posttraumatic stress disorder (PTSD) in a refugee settlement. In a randomized controlled dissemination trial in Uganda with 277 Rwandan and Somalian refugees who were diagnosed with PTSD the authors investigated the effectiveness of psychotherapy administered by lay counselors. Strictly manualized narrative exposure therapy (NET) was compared with more flexible trauma counseling (TC) and a no-treatment monitoring group (MG). Fewer participants (4\%) dropped out of NET treatment than TC $(21 \%)$. Both active treatment groups were statistically and clinically superior to $\mathrm{MG}$ on PTSD symptoms and physical health but did not differ from each other. At follow-up, a PTSD diagnosis could not be established anymore in 70\% of NET and 65\% TC participants, whereas only 37\% in MG did not meet PTSD criteria anymore. Short-term psychotherapy carried out by lay counselors with limited training can be effective to treat war-related PTSD in a refugee settlement.
\end{abstract}

Keywords: posttraumatic stress disorder, therapy, dissemination, war, refugees

In 2004, political scientists counted more than 42 ongoing wars and armed conflicts worldwide, almost all of them in developing countries (Schreiber, 2005). In addition, many low-income countries continue to suffer the consequences of recent armed conflicts. Observers of current wars have noted that the main target of the warring parties is the civilian population and that systematic atrocities including massacres and bombings are often carried out as rational strategies (Kaldor, 1999). As a result, high and sustained levels of traumatic stress affect both the mental health of individuals and the functioning of whole communities. One of the most prevailing consequences of traumatic events is postraumatic stress disorder (PTSD). Recent epidemiologic community surveys in war-torn areas found PTSD point prevalence rates between $15 \%$ and 50\% in different refugee populations (de Jong et al., 2001; Karunakara et al., 2004), in Afghanistan (Scholte et al., 2004) and

Frank Neuner, Verena Ertl, Michael Odenwald, Elisabeth Schauer, and Thomas Elbert, Department of Clinical Psychology, University of Konstanz, Konstanz, Germany, and vivo Germany, Allensbach, Germany; Patience Lamaro Onyut, vivo Uganda, Mbarara, Uganda, and Department of Science, Mbarara University of Science and Technology, Mbarara, Uganda.

This research was supported by Deutsche Forschungsgemeinschaft (DFG) and Bundesministerium für wirtschaftliche Zusammenarbeit (BMZ). We thank Mary Moran for training and treatment supervision and Silke Gotthardt and Martina Ruf for assistance with clinical examinations.

Correspondence concerning this article should be addressed to Frank Neuner, Department of Psychology, University of Konstanz, 78457 Konstanz, Germany. E-mail: frank.neuner@uni-konstanz.de
Sudan (Karunakara et al., 2004), and in countries with a history of war like Rwanda even 8 years postconflict (Pham, Weinstein, \& Longman, 2004).

Despite increasing evidence that PTSD is a major mental health issue in many developing countries and refugee populations, intervention research on these populations has been largely neglected. Treatment studies in industrialized countries have shown that PTSD caused by traumatic events like sexual violence, car accidents, criminal attacks, and so forth can be well treated through psychotherapy (Bradley, Greene, Russ, Dutra, \& Westen, 2005). Trauma-focused approaches are currently considered to be the most effective methods for the treatment of PTSD and are recommended over symptom-oriented psychotherapy or pharmacological treatment (National Institute for Clinical Excellence, 2005). Trauma-focused psychotherapy encompasses a variety of approaches including cognitive-behavioral methods that directly target the traumatic event in psychotherapy. In general, measures include encouraging the patient to talk about the traumatic event in detail and to relive the experience in a safe environment.

As the context in war-affected populations in developing countries is different from the situation in industrialized countries, it is unclear to what extent knowledge about PTSD theory and treatment can be transferred to developing countries. Many victims of war-related trauma continue to live in unstable and unsafe living conditions, affected by continuing threat, malnutrition, and poverty. It is not implausible to argue that this can interfere with the effectiveness of psychological treatment. In addition, survivors often report a series of traumatic experiences rather than a single event (Neuner, Schauer, Karunakara, et al., 2004), which makes it 
difficult to select only one significant event as the treatment focus. However, the major challenge for any mental health provisioning is the large gap between the high numbers of individuals in need of psychological assistance in the affected communities and the lack of qualified mental health professionals or counselors.

This implies the necessity of developing community-based treatment approaches that are suitable for the requirements of the field conditions in war-affected societies. The procedures have to be culturally appropriate, short, and pragmatic enough so that they can be easily disseminated to professionals with little or no training in mental health or counseling. In addition, treatment must address the needs of victims of human rights abuses and political violence and be able to deal with the effects of multiple traumatic stressors.

With these requirements in mind, we developed narrative exposure therapy (NET; Neuner, Schauer, Elbert, \& Roth, 2002 Schauer, Neuner, \& Elbert, 2005) as a standardized short-term approach that is based on the principles of cognitive-behavioral exposure therapy by adapting the classical form of exposure therapy to meet the needs of traumatized survivors of war and torture. Instead of defining a single event as a target in therapy, the patient constructs a narration about his whole life from birth up to the present situation while focusing on the detailed exploration of the traumatic experiences. As with the tradition of testimony therapy (Cienfuegos \& Monelli, 1983), the resulting written documents can be used for human rights purposes. In a randomized controlled trial (Neuner, Schauer, Klaschik, Karunakara, \& Elbert, 2004) it could be shown that NET applied by professional Western therapists was well accepted by Sudanese refugees living in a Ugandan refugee settlement and that four sessions of NET had a significant and lasting impact on the reduction of PTSD symptoms.

NET is a strictly manualized approach. Previous experiences with treatment dissemination have indicated that it is difficult to maintain strict adherence to a manual once the close supervision within a treatment trial is terminated. Since we aimed at the development of a sustainable treatment practice, we included a treatment group to simulate how NET might be used out of a trial setting in a regular care system that allowed counselors to be flexible in their treatment and to apply a variety of methods. We refer to this treatment strategy as trauma counseling (TC). For this purpose, the trainee group was trained in NET as well as in a variety of basic counseling tools, including exposure procedures, that have proven useful in a local counseling institution. The lay counselors were encouraged to follow their own intuition in the application of the treatment for an individual patient in TC.

The goal of the present trial was to examine whether trauma treatment can be effectively disseminated to lay counselors chosen from the selected refugee community itself. We used the most stringent criterion for successful treatment dissemination by testing whether the therapies conducted by the trained counselors themselves were effective. As we aimed at testing the effectiveness of trauma treatment in natural conditions, we decided to choose a refugee camp in a low-resource country as the location for the trial. While increasing external validity with this approach we had to take several risks and uncertainties into account. In particular, unpredictable political events could lead to sudden waves of migration, which would interfere with a long-term observation of study participants.
The treatment study was carried out for Rwandan and Somalian refugees in the Nakivale refugee camp in southern Uganda. We selected refugees without prior training in medical, psychological, or social professions from these communities to be trained as lay counselors for their own people. In a randomized controlled trial we compared the decline of symptoms after six sessions of NET versus six sessions of TC or a no-treatment monitoring group (MG). The main outcome measure was the score of posttraumatic stress symptoms, assessed by trained interviewers chosen from the refugee community, and expert diagnosis of PTSD at 9-months follow-up. As there is strong evidence that PTSD correlates with impaired physical health (Schnurr \& Green, 2003) and that disclosure of stressful events can improve physical symptoms (Pennebaker, 1997), we applied a scale of physical symptoms as an additional outcome measure with the hypothesis that trauma treatment improves physical health.

We expected a superior development of the two active treatment groups in comparison to the MG group at follow-up. We had no specific hypothesis for the comparison of NET and TC, as we could not predict whether the strict manual would be an advantage or an unnecessary limitation for the trained counselors.

\section{Method}

\section{Site and Population}

The Nakivale refugee settlement is one of eight official refugee camps in Uganda. At the time the study was carried out, Nakivale was host to 14,400 refugees - 12,000 Rwandan refugees from the conflicts in the early 1990s, 800 Somalians who fled to Uganda via Kenya, and several small groups of refugees from other countries. The Ugandan government and the Red Cross provided limited basic health care in the settlement. The United Nations High Commissioner for Refugees provided a basic food package of $5 \mathrm{~kg}$ beans, $10 \mathrm{~kg}$ maize meal, and $5 \mathrm{~L}$ cooking oil monthly for each registered refugee household. The refugees had to supplement this food provision themselves, mostly by farming the pieces of land that each registered refugee household had been allotted.

Permission to carry out the study was obtained from the Office of the Prime Minister, Uganda. The ethical review boards of the University of Konstanz in Germany and the Mbarara University of Science and Technology in Uganda had approved the study protocol. During the preinquiry phase of the study, the communities and their leaders were informed in meetings and supported the proposed study.

\section{Assessment}

A team of 12 research assistants was recruited from the refugee community for an epidemiological study that preceded this trial, half of them Somalians and the other half Rwandans. They were trained in a 6-week course in principles of quantitative data collection and interviewing techniques. All instruments were translated into Af-Somali and Kinyarwanda through several steps of translations, blind back-translations, and subsequent corrections by independent groups of translators. Because of a high illiteracy rate, all instruments (except for the expert evaluation) were administered in the form of standardized interviews by the trained local team. Details of training and translation of the instruments 
are described elsewhere (Onyut et al., 2004). After completing the translations, we investigated the psychometric properties of the translated scales in a validation study including a retest spanning a 2-week period and a cross-validation with expert rating. Details of this validation study will be presented elsewhere.

Sociodemographic data and trauma exposure. A previously developed sociodemographic survey (Karunakara et al., 2004) was used to assess nutritional, educational, and socioeconomic as well as displacement and general demographic information. Nutrition was assessed by asking for the number of meals eaten the previous day and by listing the various food items consumed. Since the refugees rarely have a steady income flow, their economical status was ascertained by counting the number of essential household assets such as blankets, mattresses, cooking pots, and water containers. In analysis, the items were then weighted according to current market prices in Uganda. Educational achievement was indicated by the number of years of schooling completed. For the assessment of traumatic experiences, a checklist of 31 different types of traumatic events was administered (Karunakara et al., 2004). Each event was scored as present or not present in the respondent's life. The number of different experienced and witnessed types of traumatic events was used for the estimation of the severity of trauma exposure.

Outcome measures. The Posttraumatic Stress Diagnostic Scale (PDS; Foa, 1995) is a widely used instrument for the assessment of PTSD diagnosis and severity. It consists of a frequency rating of each of the 17 criteria of PTSD according to the Diagnostic and Statistical Manual of Mental Disorders (4th ed.; American Psychiatric Association, 1994); each item is scored between 0 and 3 . The wording of the PDS items had to be adapted during the translation process to ensure semantic equivalence across the languages. In our translation, the sum score reached a retest reliability of $r_{\mathrm{tt}}=.87$ over a 2-week period. Rather than relying on a cutoff criterion, we established the diagnosis of PTSD according to the fulfillment of the $D S M-I V$ criteria assessed through the corresponding items. In a previous validation study in the same population, the PDS had reasonable diagnostic utility against an expert PTSD diagnosis that was based on the respective section of the Composite International Diagnostic Interview (CIDI; World Health Organization, 1997), with a sensitivity of .86 and a specificity of $.88(\kappa=.74 ; p<.001 ; N=98)$. Physical health was assessed with a checklist of those six illnesses and symptoms that had been present in more than $25 \%$ in the previous survey (cough, diarrhea, flu, pain, fever, and headache). The physical symptom score was calculated as the sum of symptoms/ diseases present during the last 4 weeks.

Expert evaluation. The DSM-IV diagnosis of PTSD was assessed with the PTSD part of the CIDI. The interviewers were $\mathrm{PhD}$-level psychologists or graduate students from Konstanz University who had been trained extensively in PTSD assessment with the CIDI and other instruments in previous field or laboratory studies with refugees from various cultures. The items of the interview were translated before the interview and presented with the help of trained local translators. Further inquiries about details and examples of symptoms were made by the experts with the help of the translators to ensure a correct understanding of the symptoms and to validate the clinical significance of symptoms of PTSD.

\section{Procedure}

In order to detect a moderate effect size difference between NET and TC (between-groups difference of the standardized withingroup pre- and posttest differences; $d=0.40$ ), in this trial we would need at least 133 participants for analysis at the power level $\mathrm{p} \beta=.90$. On the basis of our experiences in refugee camps, we expected a relatively high attrition (up to $40 \%$ ) of subjects from randomization to the last follow-up session, as some participants may migrate to distant locations. As a consequence, we planned to recruit 222 participants for the active treatment groups. For the comparison with the MG group we expected a higher effect size difference of $d=0.80$, which would require 66 participants for analysis at $\mathrm{p} \beta=.90$. Given the attrition rate of $40 \%$, this would require 110 subjects for analysis. To allow randomization by alternating allocation of randomly ordered participants (see below), we decided to recruit 55 participants for the MG group.

Data collection for the study was carried out in the years 2003 and 2004. The study followed an epidemiological investigation that also served as a screening procedure for the present trial. Within the survey, 1,422 refugees (906 Rwandans and 516 Somalians) were randomly chosen using a clustered sampling. The same set of instruments was used for this trial. Of the 884 refugees who fulfilled $D S M-I V$ criteria of PTSD (assessed with the PDS), we chose for this trial those 277 individuals who lived in two villages closest to the research base in the settlement.

We defined drug abuse (assessed using a checklist of common drugs in Nakivale, including Khat and Waragi), obvious mental retardation, and psychosis as exclusion criteria; however, none of the refugees approached for the trial was excluded because of these reasons. The participants were assigned to the groups in the following way: The list of participants was ordered randomly; the first 4 were consecutively assigned to the NET, TC, NET, and TC groups; and the fifth was assigned to the MG (monitoring) group. This procedure was repeated until all 277 participants were assigned.

Participants were approached by their counselors at their homestead and informed about the study protocol. If participants agreed, they signed an informed consent that was read and explained in detail to them. They were not informed about their group allocation before consenting. All refugees who were approached agreed to participate. Participants who were allocated to the MG group were informed that they would have the chance to receive the superior treatment in case the trial showed the effectiveness of at least one therapy.

The participants who received treatment were reassessed 3 and 6 months later by the same local research assistants who had carried out the interviews for the survey. They were blind with respect to the particular treatment condition. As we wanted to avoid too many assessments without offering assistance, the participants in the MG group were retested at the 6- and 9-months time points only. At 9 months after baseline the psychopathological status of the participants in all three groups was assessed by a group of five expert interviewers who were not involved in any other scientific or clinical task in the trial and who were blind with respect to the particular treatment provided. All assessment took place at the participants' homes or at a quiet place nearby. 


\section{Intervention}

Lay counselors. Nine refugees (five women, four men, mean age 27 years) from the community were trained as counselors. Skills required to be accepted for the training included literacy in English and literacy in their mother tongue, as well as the ability to empathize with their clients and a strong motivation to carry out this work. Their educational level varied from primary school $(n=$ 1) and secondary school $(n=7)$ to university education $(n=1)$. Four counselors were unemployed before the training, four were gainfully occupied, and one had been a student but her schooling had been interrupted. Three of the trainees had lifetime PTSD, and two had current PTSD. These five trainees received individual NET treatment by the trainers as part of their education.

The lay counselor group of trainees was educated in a 6-week course in general counseling skills (e.g., active listening, empathy, verbalization, emotional processing, etc.) as well as specific abilities and methods that were needed for both treatment approaches. Each trainee was educated in all approaches. The trainers were five postdoctoral- and doctoral-level personnel from the University of Konstanz, Germany, the aid organization vivo, and Mbarara University of Science and Technology, in Uganda; four of them had extensive training in trauma treatment for therapists from different cultural settings. A senior expert counselor and head of a Ugandan counseling institute based in Mbarara developed the TC protocol and guided the facilitation of training for this part of the counselors' education. Trainee counselors were then closely tutored under supervision for both approaches before beginning to work autonomously with clients. Case and personal supervision were still maintained thereafter on a weekly basis. Treatment adherence was monitored by case discussions in supervision meetings, by the direct observation of treatment sessions, and by a review of the testimonies and treatment protocols. No major deviation from the study protocol was noticed.

Treatment format. Both treatment types were conducted for six sessions (usually two sessions per week) that were between 1 and $2 \mathrm{hr}$ each in length. At the end of NET, the patient received a written record of his autobiography in his native language. After $\mathrm{TC}$, the client received a written case summary of his or her current or past problems that were discussed with the counselors-and possible solutions or coping strategies explored-also in his or her mother tongue. In each protocol the first session always included psychoeducation about the nature and prevalence of PTSD symptoms, as well as what the treatment would entail. A standard written rationale was developed for this. The goal of the procedure was to explain that PTSD-related symptoms and dysfunction were often consequent to multiple traumatic experiences.

Narrative exposure therapy. NET was carried out as outlined in the manual (Schauer et al., 2005). In NET, the participant constructs a detailed chronological account of his own biography in cooperation with the counselor. The autobiography is recorded by the counselor and corrected with each subsequent reading. Special focus of the therapy is on the transformation of the generally fragmented report of traumatic experiences into a coherent narrative. During the discussion of traumatic experiences, the counselor asks for current emotional, physiological, cognitive, and behavioral reactions and probes for respective observations. The participant is encouraged to relive these emotions while reporting the events. The discussion of a traumatic event is not terminated until a habituation of the emotional reactions presented and reported by the patient takes place. In the last session, the participant receives a written report of his biography.

Trauma counseling. To simulate how the counselors would use their skills once the strict treatment adherence control was terminated we introduced a flexible treatment approach as a control condition. We refer to this strategy as trauma counseling (TC), a combination of a variety of treatment and counseling methods that could be applied for different cases at the discretion of the therapist. The principles of TC were developed within this study and resulted from discussions with therapist trainees after their first training treatments. Further input was received from an experienced Ugandan senior counselor who assisted in training and supervision within this trial. TC was less directive then NET and more oriented toward the psychological and social needs expressed by the individual client. In particular, the discussion of current life problems and conflicts was allowed as part of the treatment. Narrative exposure of traumatic events was considered to be a part of TC but not mandatory. However, a main principle of TC was to relate current problems to past traumatic experiences. Additional skills that were taught for TC included nondirective active listening, problem solving, the exploration of coping skills, and grief interventions.

\section{Analysis}

Baseline characteristics of the groups were compared using chi-square tests for categorical variables and analyses of variance for continuous variables to examine the effects of randomization. To seek any systematic effect on dropouts and missing data, we also compared treatment completers with dropouts on baseline variables (chi-square tests and $t$ tests), and we compared participants with complete data with those who were missing data.

Aiming at an intention-to-treat analysis, we included in the outcome analysis all participants who were randomized. In anticipation of a high rate of missing data, we considered a lastobservation-carried-forward procedure as too conservative. Instead we chose to apply mixed-effects models that allow the inclusion of all available data without the arbitrary replacement or imputation of missing values. Recent simulation studies have indicated that mixed-effects models are more accurate than statistical multiple imputation strategies in dealing with missing data (Fong, Rai, \& Lam, 2006).

For the outcome variables PDS and health scores, we calculated multilevel mixed-effects models including participant as random effect and nationality, treatment, time, Nationality $\times$ Treatment, and Treatment $\times$ Time as fixed effects, whereby participant was nested in treatment and nationality. Following our previous treatment study (Neuner, Schauer, Klaschik, et al., 2004), we expected a decrease of symptoms from pretest to posttest and a further decrease from posttest to follow-up. Consequently, we used linear models rather than a curvilinear trend to estimate time effects.

As a mixed-effects model makes use of all data available, we did not replace any missing data using a last-observation-carriedforward strategy. Each active treatment group was compared with the MG group using a contrast analysis of the Time $\times$ Treatment interaction. Physical health was additionally compared using chisquare tests on the presence of single symptoms and diseases at follow-up. Clinical significance was estimated by calculating 
within-treatment effect sizes (Cohen's $d$; difference between means of an outcome measure at two time points divided by the pooled standard deviation for these means) for PTSD and health outcome. In addition, the diagnostic status of individuals at the expert evaluation was compared between the groups using a chisquare test.

Data analysis was carried out with JMP 6, which allows multilevel mixed-effects models including contrast analysis based on the SAS PROC MIXED procedure.

\section{Results}

\section{Baseline Characteristics}

Baseline characteristics of the treatment groups are presented in Table 1. Randomization resulted in a significant difference in the ratio of nationalities in the groups. Consequently, nationality was used as additional factor in the outcome analyses.

\section{Dropouts and Missing Data}

In the NET group, 4 participants (3.6\%) refused or dropped out of treatment, whereas in the TC group 22 participants (19.8\%) did not complete therapy (see flow chart in Figure 1). The difference was significant, $\chi^{2}(1, N=222)=14.12, \mathrm{p}=<.001$. Dropouts/ refusers did not differ significantly from treatment completers in age, nationality, possessions, number of event types, or pretest PDS and health scores (all $p s>.20$ ). Figure 1 presents the number of participants who could not be followed up at the different time points. The comparison of baseline characteristics between followed-up participants with complete data and participants with missing data using $t$ tests for continuous and -chi-square tests for categorical variables did not result in any significant difference in age, nationality, possessions, number of event types or pretest PDS and health scores (all $p s>.20$ ).

\section{The PDS Score}

Results on the outcome variables are presented in Table 2. For the PDS sum score, the two active treatment groups were compared over time, including all time points. A mixed-effects model was calculated with the PDS sum score as an dependent variable; participant as random effect; and treatment, time, nationality, Time $\times$ Treatment, and Nationality $\times$ Treatment as fixed effects. There was a significant main effect of time, $F(2,254)=292.7$, $p<.001$, and nationality (with the Somalians showing higher symptom severity than did the Rwandans), $F(1,220)=55.8, p<$ .001 . The Nationality $\times$ Treatment and Time $\times$ Treatment interactions were not significant, $p=.87$ and .63 , respectively. A contrast analysis using a Bonferroni-Holm adjustment of significance level revealed that participants improved from pretest to posttest, $F(1,254)=463, p<.001$, but posttest and follow-up did not differ significantly, $F(1,254)=0.4, p=.60$.

To test the hypothesis of a superiority of both treatment groups in comparison to the $\mathrm{MG}$ group, we performed a further analysis using the PDS sum score as dependent variable. For the MG group, data was available for the time points pretest and the follow-up; consequently we analyzed a model restricted on these two time points. A mixed-effects model was calculated using participant as random effect and treatment, time, nationality, Time $\times$ Treatment, and Nationality $\times$ Treatment as fixed effects. There were significant main effects of time, $F(1,112)=188.7, p<.001$, and nationality (with the Somalians showing higher symptom severity than did the Rwandans), $F(1,277)=48.0, p<.001$, but Nationality $\times$ Treatment was not significant, $p=6$. The Time $\times$ Treatment effect was significant, $F(2,112)=6.03, p<.01$. In a contrast analysis of the Time $\times$ Treatment interaction, each active treatment group was compared with the MG group. Using a Bonferroni-Holm correction of the significance level, TC was

Table 1

Baseline Sociodemographic Characteristics of Participants Divided by Groups

\begin{tabular}{|c|c|c|c|c|}
\hline Characteristic & $\operatorname{NET}(n=111)$ & $\mathrm{TC}(n=111)$ & $\mathrm{MG}(n=55)$ & $p$ \\
\hline Gender: $n(\%)$ & & & & .92 \\
\hline Men & $55(49.5)$ & $52(46.8)$ & $28(50.9)$ & \\
\hline Nationality: $n(\%)$ & & & & $<.01$ \\
\hline Somalian & $61(67.7)$ & $52(46.8)$ & $12(21.8)$ & \\
\hline Rwandan & $50(32.3)$ & $59(53.2)$ & $43(79.1)$ & \\
\hline Age in years: $M(S D)$ & $34.4(12.2)$ & $35.2(12.8)$ & $35.6(14.0)$ & .85 \\
\hline Education in years: $M(S D)$ & $4.38(4.13)$ & $4.43(4.29)$ & $3.25(3.31)$ & .17 \\
\hline Occupation: $n(\%)$ & & & & .12 \\
\hline None & $60(54.1)$ & $56(50.4)$ & $19(34.5)$ & \\
\hline Farmer & $24(21.6)$ & $26(32.4)$ & $29(52.7)$ & \\
\hline Household & $15(13.5)$ & $14(12.6)$ & $4(7.2)$ & \\
\hline Student & $3(2.7)$ & $4(3.6)$ & $1(1.8)$ & \\
\hline Other & $9(8.1)$ & $11(9.9)$ & $2(3.6)$ & \\
\hline Marital status: $n(\%)$ & & & & .60 \\
\hline Married & $78(70.2)$ & $67(60.3)$ & $32(58.2)$ & \\
\hline Single & $23(20.7)$ & $30(27.0)$ & $16(29,1)$ & \\
\hline Widowed & $6(5.4)$ & $8(7.2)$ & $6(10.9)$ & \\
\hline Separated & $4(3.6)$ & $6(5.4)$ & $1(1.8)$ & \\
\hline No. of meals/day: $M(S D)$ & $1.26(0.48)$ & $1.31(0.53)$ & $1.42(0.53)$ & .18 \\
\hline Value of possessions in US\$: $M(S D)$ & $9.07(11.6)$ & $9.56(13.8)$ & $10.7(13.4)$ & .73 \\
\hline No. of traumatic event types: $M(S D)$ & $14.5(4.96)$ & $14.1(5.63)$ & $13.1(4.44)$ & .27 \\
\hline
\end{tabular}

Note. $\quad \mathrm{NET}=$ narrative exposure therapy; $\mathrm{TC}=$ trauma counseling; $\mathrm{MG}=$ monitoring group. 


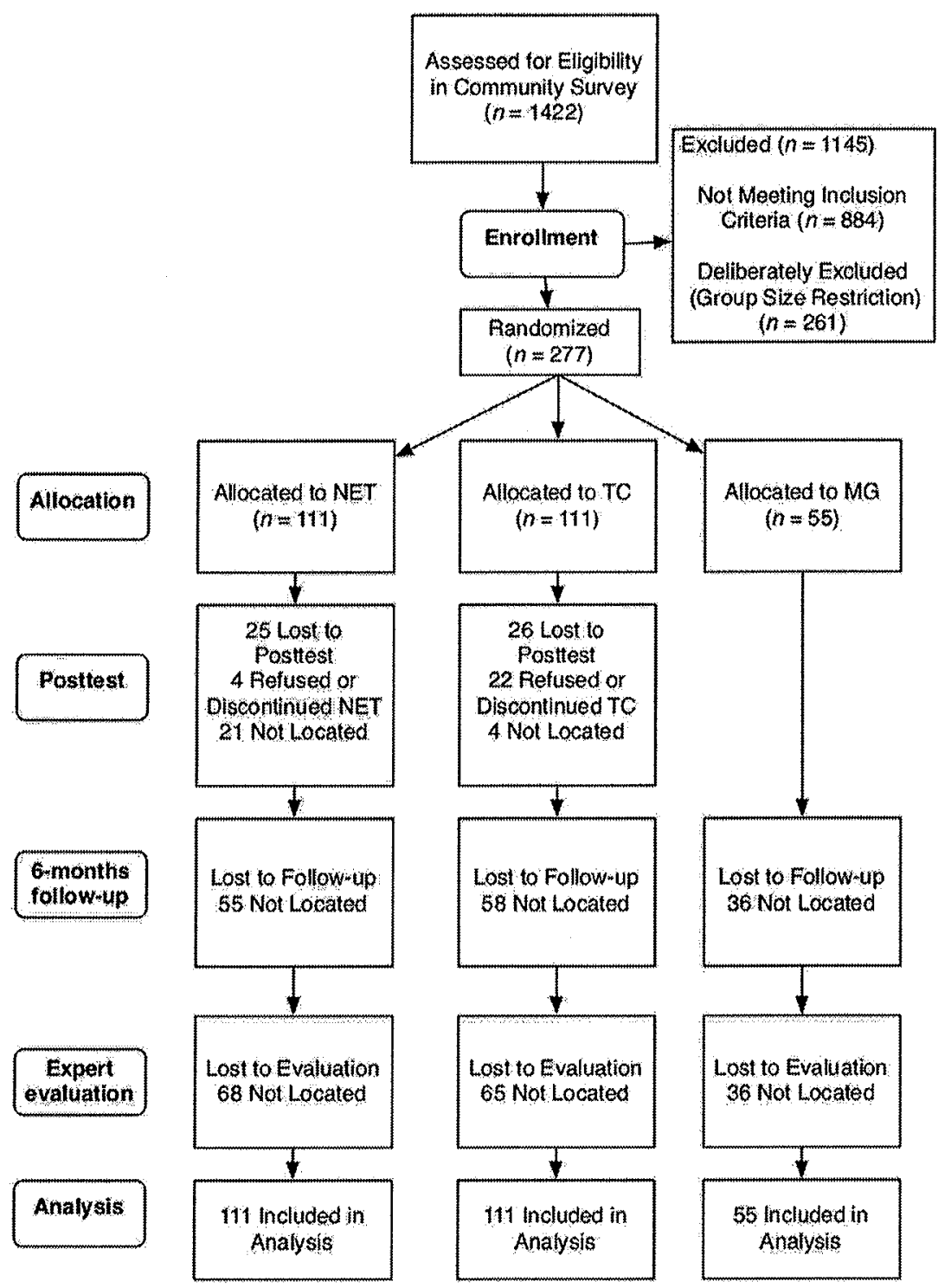

Figure 1. Flow chart of the study protocol.

superior to the MG group, $F(1,112)=8.2, p=.0008$. The same was true for NET, $F(1,112)=8.2, p=.005$.

\section{Clinical Significance}

To estimate the clinical significance of treatment effects, we examined the PTSD diagnoses made by experts at follow-up (see Figure 2). In the NET group, $69.8 \%(n=30)$ of participants no longer fulfilled the criteria for PTSD, and in the TC group, 65.2\% no longer had PTSD whereas in the MG group, only $36.8 \%$ were free of diagnosis. A chi-square test showed differences between the three treatment groups to be generally significant, $\chi^{2}(2, N=$ $108)=6.3, p<.042$. Both active treatment groups were compared with the MG group, and the significance level was adjusted according to Bonferroni-Holm. The difference between NET and
MG was significant, $\chi^{2}(1, N=53)=5.7, p=.017$, as was the difference between TC and MG, $\chi^{2}(1, N=65)=4.4, p=.036$.

\section{Physical Health}

To estimate the changes in physical health symptoms, we calculated a sum score of the number of physical health symptoms experienced in the last year for the baseline and the follow-up time point. In a mixed model including the factors participant as random effect and treatment, time, nationality, Time $\times$ Treatment, and Nationality $\times$ Treatment as fixed effects, there were significant main effects of nationality, $F(1,116)=6.60, p=.01$, as well a significant interaction of Time $\times$ Treatment, $F(2,116)=4.60$, $p=.01$. In a contrast analysis of the Time $\times$ Treatment interaction, TC was superior to the MG group, $F(1,112)=5.56, p=.02$, 
Table 2

Means (SDs) and Within-Treatment Effect Sizes (Cohen's d) of the Outcome Variables by Group

\begin{tabular}{|c|c|c|c|c|c|}
\hline Treatment group & Pre & Post & Follow-up & $d$ (pre to post) & $d$ (pre to follow-up) \\
\hline \multicolumn{6}{|c|}{ Posttraumatic Stress Diagnostic Scale } \\
\hline NET & $25.9(13.2)$ & $5.4(6.6)$ & $6.1(6.8)$ & 1.4 & 1.4 \\
\hline $\mathrm{TC}$ & $26.7(12.5)$ & $5.3(5.7)$ & $5.0(6.6)$ & 1.5 & 1.5 \\
\hline MG & $21.3(10.6)$ & - & $10.1(8.1)$ & - & 0.8 \\
\hline \multicolumn{6}{|c|}{ Physical symptom score } \\
\hline NET & $3.1(1.8)$ & $2.8(1.6)$ & $1.8(1.7)$ & 0.1 & 0.9 \\
\hline $\mathrm{TC}$ & $3.3(1.8)$ & $2.9(1.8)$ & $2.3(1.6)$ & 0.2 & 0.5 \\
\hline MG & $3.1(1.8)$ & - & $3.6(1.7)$ & - & -0.2 \\
\hline
\end{tabular}

Note. Dashes signify that data were not collected. Pre $=$ pretest; Post $=$ posttest NET $=$ narrative exposure therapy; $\mathrm{TC}=$ trauma counseling; $\mathrm{MG}=$ monitoring group.

and NET was superior to MG, $F(1,116)=9.17, p<.01$. To examine potential mechanisms of this effect, we analyzed the treatment effect on single symptoms. For this exploratory analysis, we calculated chi-square test for the pretest and the follow-up prevalence rates of each symptom. Results of this calculation are illustrated in Figure 3. A significant treatment effect was found for cough, diarrhea, and fever, whereas the effect on flu, pain, and headache was not significant.

\section{Discussion}

We carried out a randomized controlled dissemination study of psychotherapy for PTSD in a refugee settlement in Uganda. With few exceptions, psychotherapy intervention research involving randomized trials in resource-poor countries is almost absent, although epidemiological studies indicate a high need for treatment (Patel, 2007). We found that over the period of 6-9 months, the groups that received active treatment developed statistically and clinically better on scales of PTSD than did a nontreated monitoring group.

Although the net effect size was smaller in this trial in comparison to the previous Imvepi study (Neuner, Schauer, Klaschik, et

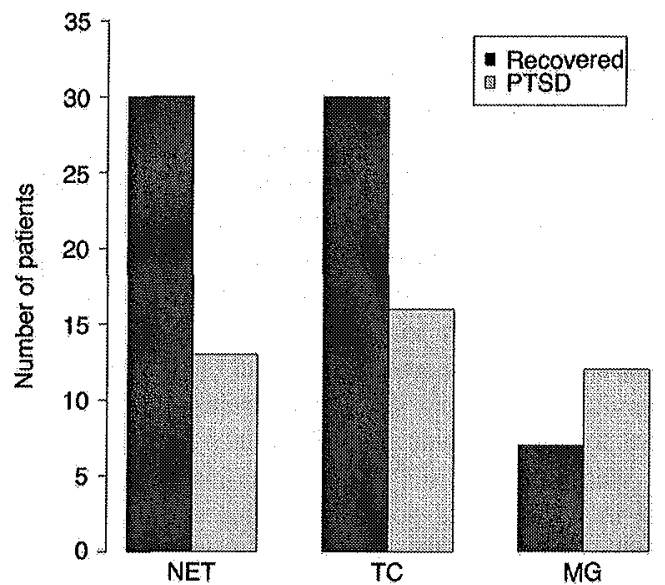

Figure 2. Diagnostic status of participants at 9-months follow-up, divided by treatment group. al., 2004) where NET was carried out by expert therapists, the results indicate that it is possible to treat war-related PTSD in refugee populations and that effective psychotherapy can be carried out by trained lay counselors after only 6 weeks of training. We could also show that physical health improved through psychotherapy. Although there have been indications that psychological interventions, especially the disclosure of stressful events, can improve physical symptoms (Pennebaker, 1997), it is noteworthy and of public health interest that such an effect can be found in a refugee camp under deficient hygienic and health care conditions.

In contrast to results of the first trial in the Imvepi settlement (Neuner, Schauer, Klaschik, et al., 2004), a decrease of symptoms was also observed in the nontreated monitoring group in the current study. This improvement, in mental but not somatic symptoms, might be attributed to regression to the mean or spontaneous remission, as the living conditions, including the nutritional situation, was much more stable in Nakivale than in the Imvepi camp. However, the spontaneous improvement reached clinical significance for only a minority of the participants.

The comparison of NET and TC showed no significant difference between the approaches in reducing PTSD symptoms. However, significantly fewer participants dropped out of the NET treatment. One reason might be the perceived value of the written NET testimony, which participants receive at the end of treatment. As this study was designed as a pragmatic dissemination study rather than a dismantling trial, the mechanisms of the treatment effects cannot be identified. However, as supportive counseling without a trauma exposure component was less effective than NET in a previous study (Neuner, Schauer, Klaschik, et al., 2004), it could be argued that the encouragement to address the traumatic event in treatment might be an essential element of effective trauma treatment, regardless of whether treatment is carried out in a usual health care system or in the conditions of a refugee settlement. At the same time, the application of additional counseling techniques did not increase efficacy of treatment, which fits well to the common finding that the combination of treatment approaches does not enhance efficacy in PTSD psychotherapy (Foa et al., 1999, 2005; Marks, Lovell, Noshirvani, Livanou, \& Thrasher, 1998). Alternatively, factors like treatment credibility and therapist motivation might have affected dropout rates. 

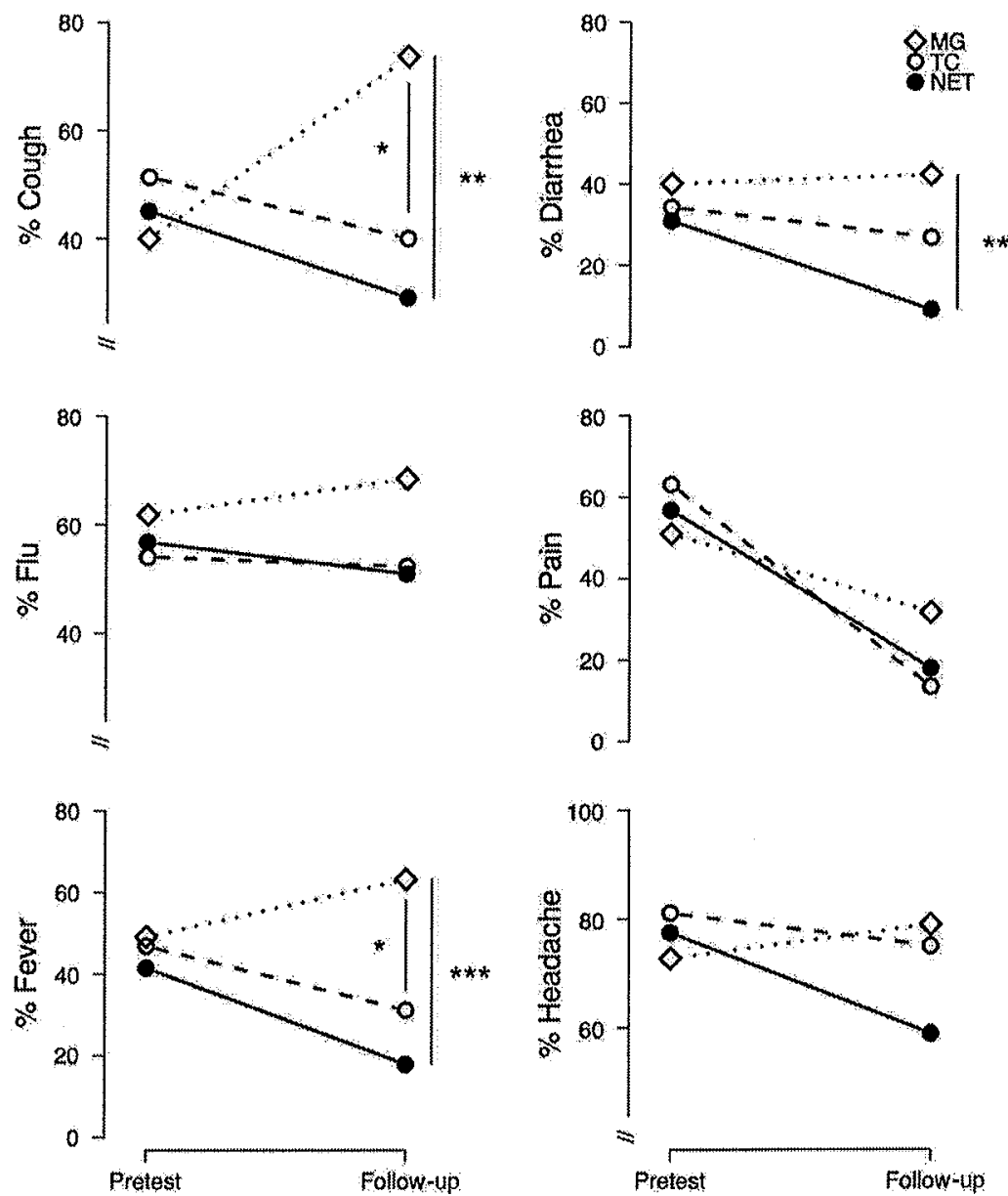

Figure 3. Baseline and 6-months follow-up prevalence rates of physical symptoms and diseases, divided by

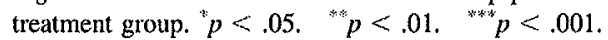

Although we aimed at carrying out a trial with rigorous scientific methods, we could not prevent some limitations. First of all, a high number of participants could not be located for follow-up examinations. We could not receive reliable information about the destination of all participants we could not locate. One explanation for the high attrition might be that rumors about a forced repatriation have stimulated hiding among the Rwandan refugees. At the same time, a resettlement program had started for the Somalian refugees, allowing them to migrate to Western countries, which caused substantial migration from the settlement mainly to the United States. Although we could not detect any significant baseline difference between individuals with complete data and those with lost data, it is possible that an unknown systematic effect has caused selective dropout from the study. Participants with missing data might have improved either more or less than did the participants who could be reexamined. As a result, the treatment effects noted in this study might be over- or underestimated. However, the finding that the treated refugees improved more than did the nontreated refugees would be compromised only if the effect went in opposite directions for the treated and nontreated groups, which is implausible though not impossible. Secondly, due to unknown reasons and despite a careful procedure, randomization resulted in a significantly different group composition with regard to nationality. As the inclusion of nationality as a factor in the analysis did not interfere with the findings, we have no reason to assume that the results were seriously compromised by this confound.

Another limitation of the study was the concentration on PTSD and physical health. As the translation and validation of useful instruments takes a huge effort, we decided to concentrate on the quality of restricted measurements rather than include many ad hoc translated scales. Further trials should include measurements of depression and more elaborate assessment of functioning.

To summarize, we could show that within a refugee settlement short-term trained refugees can effectively treat their neighbor refugees who suffer from PTSD. Regarding the high need for treatment in war-affected populations, this approach might give one possible answer to urgent mental health problems of waraffected populations. However, due to the limitations of the study, in particular the high attrition of participants during the course of the trial, these findings have to be interpreted carefully. Migration 
is a factor that might interfere with any longitudinal study with refugees. As a consequence, the results of large-scale treatment studies have to be complemented by smaller trials that managed to track the refugees who have migrated during the course of the study (Neuner, Schauer, Klaschik, et al., 2004), as well as larger trials with trained local counselors in more stable populations in resource-poor countries (Bolton et al., 2003). Taken together, these studies indicate that evidence-based psychotherapy can be feasible and effective within developing countries and that treatment approaches can be disseminated to local counselors. In addition, these studies demonstrated that intervention research is possible and warranted in developing countries and crisis regions.

\section{References}

American Psychiatric Association. (1994). Diagnostic and statistical manual of mental disorders (4th ed.). Washington, DC: Author.

Bolton, P., Bass, J., Neugebauer, R., Verdeli, H., Clougherty, K. F., Wickramaratne, P., et al. (2003). Group interpersonal psychotherapy for depression in rural Uganda: A randomized controlled trial. Journal of the American Medical Association, 289(23), 3117-3124.

Bradley, R., Greene, J., Russ, E., Dutra, L., \& Westen, D. (2005). A multidimensional meta-analysis of psychotherapy for PTSD. American Journal of Psychiatry, 162(2), 214-227.

Cienfuegos, J., \& Monelli, C. (1983). The testimony of political repression as a therapeutic instrument. American Journal of Orthopsychiatry, 53, $43-51$.

de Jong, J. T. V. M., Komproe, I. H., Van Ommeren, M., El Masri, M., Araya, M., Khaled, N., et al. (2001). Lifetime events and postraumatic stress disorder in 4 postconflict settings. Journal of the American Medical Association, 286(5), 555-562.

Foa, E. B. (1995). Posttraumatic Stress Diagnostic Scale [Manual]. Minneapolis, MN: National Computer Systems.

Foa, E. B., Dancu, C. V., Hembree, E. A., Jaycox, L. H., Meadows, E. A., \& Street, G. P. (1999). A comparison of exposure therapy, stress inoculation training, and their combination for reducing posttraumatic stress disorder in female assault victims. Journal of Consulting and Clinical Psychology, 67(2), 194-200.

Foa, E. B., Hembree, E. A., Cahill, S. P., Rauch, S. A., Riggs, D. S., Feeny, N. C., et al. (2005). Randomized trial of prolonged exposure for posttraumatic stress disorder with and without cognitive restructuring: Outcome at academic and community clinics. Journal of Consulting and Clinical Psychology, 73(5), 953-964.

Fong, D. Y. T., Rai, S. N., \& Lam, K. S. L. (2006, September). Use of multiple imputation on linear mixed model and generalized estimating equations for longitudinal data analysis: A simulation study. Paper presented at the Thirteenth International Conference of the Forum for Interdisciplinary Mathematics, Tomar: Portugal.

Kaldor, M. (1999). New and old wars: Organized violence in a global era. London: Blackwell.

Karunakara, U. K., Neuner, F., Schauer, M., Singh, K., Hill, K., Elbert, T., et al. (2004). Traumatic events and symptoms of post-traumatic stress disorder amongst Sudanese nationals, refugees and Ugandans in the West Nile. African Health Sciences, 4(2), 83-93.

Marks, I., Lovell, K., Noshirvani, H., Livanou, M., \& Thrasher, S. (1998). Treatment of posttraumatic stress disorder by exposure and/or cognitive restructuring: A controlled study. Archives of General Psychiatry, 55(4), 317-325.

National Institute for Clinical Excellence. (2005). Post-traumatic stress disorder. London: Gaskell and the British Psychological Society.

Neuner, F., Schauer, M., Elbert, T., \& Roth, W. T. (2002). A narrative exposure treatment as intervention in a Macedonian refugee camp: A case report. Journal of Behavioural and Cognitive Psychotherapy, 30 , 205-209.

Neuner, F., Schauer, M., Karunakara, U., Klaschik, C., Robert, C., \& Elbert, T. (2004, October 25). Psychological trauma and evidence for enhanced vulnerability for posttraumatic stress disorder through previous trauma among West Nile refugees. BMC Psychiatry, 4, Article 34. Neuner, F., Schauer, M., Klaschik, C., Karunakara, U., \& Elbert, T. (2004). A comparison of narrative exposure therapy, supportive counseling, and psychoeducation for treating posttraumatic stress disorder in an African refugee settlement. Journal of Consulting and Clinical Psychology, 72(4), 579-587.

Onyut, L. P., Neuner, F., Schauer, E., Ertl, V., Odenwald, M., Schauer, M., et al. (2004). The Nakivale camp mental health project: Building local competency for psychological assistance to traumatised refugees. Intervention, 2(2), 90-107.

Patel, V. (2007). Mental health in low- and middle-income countries. British Medical Bulletin, 81-82, 81-96.

Pennebaker, J. W. (1997). Writing about emotional experiences as a therapeutic process. Psychological Science, 8(3), 162-166.

Pham, P. N., Weinstein, H. M., \& Longman, T. (2004). Trauma and PTSD symptoms in Rwanda: Implications for attitudes toward justice and reconciliation. Journal of the American Medical Association, 292(5), 602-612.

Schauer, M., Neuner, F., \& Elbert, T. (2005). Narrative exposure therapy: A short term intervention for traumatic stress disorders after war, terror or torture. Göttingen, Germany: Hogrefe \& Huber.

Schnurr, P. P., \& Green, B. L. (2003). Trauma and health: Physical health consequences of exposure to extreme stress. Washington, DC: American Psychological Association.

Scholte, W. F., Olff, M., Ventevogel, P., de Vries, G. J., Jansveld, E., Cardozo, B. L., et al. (2004). Mental health symptoms following war and repression in eastern Afghanistan. Journal of the American Medical Association, 292(5), 585-593.

Schreiber, W. (2005). Das Kriegsgeschehen 2004. Daten und Tendenzen der Kriege und bewaffneten Konflikte [War events 2004: Data and tendencies of the wars and armed conflicts]. Wiesbaden, Germany: Vs Verlag.

World Health Organization. (1997). Composite International Diagnostic Interview (CIDI). Geneva, Switzerland: Author. 